\title{
Novel synthetic ROR $\gamma$ agonist compounds as a potential anti-tumor therapeutic approach
}

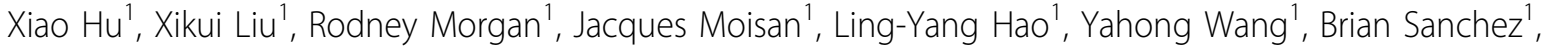 \\ Charles Lesch', Dick Bousley ${ }^{1}$, Clark Taylor ${ }^{1}$, Thomas Aicher ${ }^{1}$, Peter Toogood', Weiping Zou ${ }^{2}$ Gary Glick', \\ Laura Carter ${ }^{1 *}$
}

From Society for Immunotherapy of Cancer 29th Annual Meeting

National Harbor, MD, USA. 6-9 November 2014

\section{Introduction}

Selective enhancement (or activation) of the immune system by novel small molecules may be a potential therapeutic approach for the treatment of cancer. ROR $\gamma t$ (Retinoic Acid Receptor-related orphan receptor) is the key transcription factor for the development of $\mathrm{CD} 4^{+}$Th17 cells, CD8 ${ }^{+}$Tc17 cells and $\mathrm{IL}_{-1} 7^{+}$innate immune cells including $\gamma \delta \mathrm{T}$ cells. A member of the nuclear receptor superfamily, ROR $\gamma$ modulates the expression of cytokines, chemokines and their receptors to induce a pro-inflammatory environment. ROR $\gamma$ can interact with other lineage-associated transcription factors resulting in developmental plasticity which reinforces immunity and limits immunosuppressive mechanisms. These activities suggest that the activation of ROR $\gamma$ may enhance anti-tumor immune responses and Th17 and Tc17 cells have been reported to have potent anti-tumor effects in vivo.

\section{Results}

We have discovered a series of synthetic ROR $\gamma$ agonist compounds that enhance the activity of an ROR $\gamma$-dependent reporter and increase IL-17A, IL-17F, IL-22 and GMCSF production by murine and human T cells. $\mathrm{CD}^{+} \mathrm{T}$ cell stimulation with cytokine cocktails including TGF $\beta$ in vitro can generate Th17 and Treg cells; the addition of ROR $\gamma$ agonists decreases the expression FOXP3 while concomitantly increasing IL-17 and GM-CSF mRNA expression. In addition, stimulation of $\mathrm{T}$ cells in the presence of ROR $\gamma$ agonist compounds generates effector $\mathrm{T}$ cells that resist PD-1/PD-L1-mediated inhibition of proliferation and cytokine production. Taken together, the enhanced production of cytokines, decreased generation of Treg

'Lycera Corp., Ann Arbor, MI, USA

Full list of author information is available at the end of the article cells and resistance to PD-1 checkpoint inhibition supports ROR $\gamma$ agonists having potential anti-tumor activities. Indeed, OVA-specific $\mathrm{CD} 8^{+} \mathrm{T}$ cells activated in vitro with ROR $\gamma$ agonist compounds reduced growth of OVAexpressing EG7 and B16F10 tumors more effectively than cells stimulated without ROR $\gamma$ agonist. Flow cytometric analysis of tumors over time showed increased numbers of agonist treated cells present as tumor infiltrating lymphocyte (TILs). A higher percentage of these TILs were IL- $17^{+}$ with increased mean fluorescent intensity for IL-17. Agonist treated TILs had increased expression of CD62L and decreased expression of PD-1 suggesting that agonist treatment preserves a less differentiated, more central memory phenotype that may be less susceptible to checkpoint inhibition and have increased survival capacity.

\section{Conclusions}

ROR $\gamma$ agonist compounds enhance immune-associated antitumor pathways, decrease immunosuppressive mechanisms and induce effector $\mathrm{T}$ cells which decrease tumor growth. Immune enhancement by ROR $\gamma$ agonists may therefore represent a unique anti-tumor approach which could also complement other immunotherapy approaches.

\section{Authors' details \\ 'Lycera Corp., Ann Arbor, MI, USA. ${ }^{2}$ Dept. of Surgery, Immunology, Biology, University of Michigan, Ann Arbor, MI, USA.}

Published: 6 November 2014

doi:10.1186/2051-1426-2-S3-P194

Cite this article as: Hu et al: Novel synthetic RORy agonist compounds as a potential anti-tumor therapeutic approach. Journal for ImmunoTherapy of Cancer 2014 2(Suppl 3):P194. 